\title{
Direct activation of neutrophil chemiluminescence by rheumatoid sera and synovial fluid
}

\author{
R. GALE, J. V. BERTOUCH, J. BRADLEY, AND P. J. ROBERTS-THOMSON \\ From the Department of Clinical Immunology, Flinders Medical Centre, Bedford Park, South Australia 5042
}

SUMMARY The majority of paired sera and synovial fluids from 21 patients with rheumatoid arthritis produced a rapid chemiluminescent response when incubated with human neutrophils. Synovial fluid gave considerably higher responses than the paired serum specimen. In contrast little or no response was found with paired sera and joint fluid taken from patients with gout, psoriasis, and osteoarthritis and with sera from healthy donors. A similar chemiluminescent response was observed when neutrophils were preincubated with large aggregates of heated human gammaglobulin (HAGG), which were used as a model of immune complexes. Smaller nonreactive aggregates of gammaglobulin became reactive after preincubation with a purified monoclonal rheumatoid factor (mRF) which had a high avidity for aggregated IgG. The addition of this monoclonal rheumatoid factor also caused enhancement of chemiluminescence by rheumatoid sera. Further evidence suggesting that the active material found in these rheumatoid specimens contained complexed immunoglobulin was obtained by indirect immunofluorescence. Neutrophils developed intracellular immunoglobulin inclusions after preincubation in reactive rheumatoid sera but not with nonreactive or normal sera. However, activation of neutrophil chemiluminescence by rheumatoid specimens did not correlate significantly with levels of rheumatoid factor or immune complexes suggesting that the activating complexes were of a particular type. In conclusion we have shown the direct activation of neutrophil chemiluminescence by rheumatoid sera and synovial fluid and suggest that the activation is caused by large IgG-containing immune complexes. It is possible that this activation may have important implications in the immunopathogenesis of the rheumatoid inflammatory process.

Rheumatoid arthritis (RA) is an autoimmune disorder of uncertain immunopathogenesis, but immune complexes have been strongly implicated in many of the clinical manifestations. ${ }^{12}$ It is possible that an important component of the inflammatory processes characteristic of this disease may be the result of direct neutrophil activation during the phagocytosis of these reactive immune complexes both in the circulation and in the articular, pleural, and pericardial spaces. ${ }^{3-6}$ The resultant neutrophil activation may initiate the production and release of highly reactive superoxide free radicals which are likely candidates for causing subsequent tissue damage.

There is some evidence to support this hypothesis in RA. Neutrophils taken from patients with Felty's

Accepted for publication 12 March 1982.

Correspondence to Dr P. Roberts-Thomson. syndrome (FS) contain larger intracellular immunoglobulin inclusions and have reduced functional activity. ${ }^{34}$ Similar findings have been described following the incubation of normal neutrophils in sera from patients with RA. ${ }^{4}$

Previous studies of the interaction between serum or synovial fluid from RA and FS patients have centred on the alterations to neutrophil functional capability. ${ }^{4} 7$ In this study we have used chemiluminometry to demonstrate the direct activation of normal neutrophils by rheumatoid sera and synovial fluid and provide evidence relating to the nature of this reactive material.

\section{Materials and methods}

Samples of serum and knee synovial fluid were obtained from 31 patients with joint diseases. There were 21 patients with definite or classical rheumatoid 
arthritis (RA) defined according to the American Rheumatism Association criteria and 10 patients with other joint diseases (non-RA). This latter group consisted of patients with gout, psoriasis, and osteoarthritis. Two patients with Felty's syndrome were defined by the presence of RA in association with splenomegaly and neutropenia (leucocytes $\left.<2000 / \mathrm{mm}^{3}\left(2 \times 10^{9} / \mathrm{l}\right)\right)$. A single specimen of highly reactive pleural fluid was studied in more detail, as large volumes were available. This specimen was obtained from an elderly male patient with probable RA. His symptoms included an acute symmetrical polyarthritis with pleural and pericardial effusions. Rheumatoid factor and antinuclear antibody were both absent in the pleural fluid, but rheumatoid factor was present in the serum in low levels.

Neutrophils were prepared from preservative-free heparinised blood obtained from group $\mathrm{O}$ healthy individuals. The blood was diluted with an equal volume of phosphate buffered saline (PBS) and the mononuclear cells removed by centrifuging through Ficol-Hypaque (Pharmacia). The pellet was resuspended in double the volume of $2 \%$ dextran in PBS and allowed to stand at room temperature for 45 minutes to sediment the red cells. The supernatant containing the neutrophils was removed, and contaminating red cells were lysed by 5 minutes' incubation in Gey's solution. The preparation was adjusted to $5 \times 10^{6}$ neutrophils $/ \mathrm{ml}$ in Eagle's MEM (Flow) containing $5 \%$ fetal calf serum and maintained at $37^{\circ} \mathrm{C}$.

Luminol (3-aminophthalhydrazide, Koch Light Laboratories) stock solution was $0.056 \mathrm{M}$ in dimethyl sulphoxide. This was diluted 1:40 in MEM and prewarmed to $37^{\circ} \mathrm{C}$ before use.

Chemiluminescence $(\mathrm{CL})$ was measured in an LKB luminometer (model 1250 ) fitted with a $37^{\circ} \mathrm{C}$ water jacketed sample holder and low-speed rotation of the reaction vessel to maintain continuous mixing of the cell suspension. $200 \mu \mathrm{l}$ of neutrophil preparation was incubated at $37^{\circ} \mathrm{C}$ for $5 \mathrm{~min}$, with $100 \mu$ l of the test serum, synovial fluid, or column fractions. $600 \mu \mathrm{l}$ of luminol solution was then added and the rate of chemiluminescence activity recorded. The maximum chemiluminescent activity (CL max) was defined as the highest recorded light output (in millivolts) integrated over one-second intervals.

Heat-aggregated IgG (HAGG) was prepared by heating Cohn fraction II gammaglobulin $(160 \mathrm{mg} / \mathrm{ml}$ (160 g/l), Commonwealth Serum Laboratories), diluted $1: 8$ with $\mathrm{MEM}$ at $63^{\circ} \mathrm{C}$ for varying periods of time up to 60 minutes.

Filtration chromatography. Gel filtration was performed at room temperature with either a $90 \times 2.5$ cm Sephacryl S300 or a Sepharose 6B (Pharmacia) column with PBS pH $7 \cdot 3$ without preservative as eluant. An upward flow rate of $20 \mathrm{ml} /$ hour was maintained, and $6 \mathrm{ml}$ fractions were collected.

Indirect immunofluorescent studies were carried out on neutrophils preincubated for 1 hour in serum or synovial fluid at the same dilutions as used for chemiluminescence. The cells were washed and slides prepared with a Shandon cytocentrifuge. The slides were fixed in $3 \%$ formalin for 5 minutes, washed twice in PBS, placed in acetone at $-20^{\circ} \mathrm{C}$ for 5 minutes, and washed twice with excess PBS. Fluorescein-labelled rabbit $\mathrm{F}(\mathrm{ab})_{2}^{1}$ anti-immunoglobulin (Behring) diluted 1:2 in PBS was layered on the slide preparations and incubated at $4^{\circ} \mathrm{C}$ for 60 minutes. The slides were then washed, mounted, and examined with fluorescent microscopy under epiillumination.

Incubation with monoclonal rheumatoid factor (mRF). Serum samples or preparations of HAGG were preincubated with $10 \% \mathrm{v} / \mathrm{v}$ solution of a purified preparation of $\mathrm{mRF}(8.6 \mathrm{mg} / \mathrm{ml}(8.6 \mathrm{~g} / \mathrm{l}))$ for 60 minutes at $37^{\circ} \mathrm{C}$ before testing for neutrophil CL activity. The mRF was obtained from a patient with a lymphoproliferative disorder, and its purification and properties have been previously described. ${ }^{8}$

Other immunological tests. Immune complexes were measured by the liquid phase $\mathrm{C} 1 \mathrm{q}$ binding test, rheumatoid factor was measured by the sheep cell agglutination technique, and immunoglobulins were measured by laser nephelometry as previously described. ${ }^{9}$

\section{Results}

The maximum chemiluminescent activity ( $C L \max$ ) of the paired serum and synovial fluid samples taken from 31 patients with joint disease are shown in Fig. 1. Significantly raised levels of neutrophil chemiluminescence were seen in the serum $(p<0.05)$ and synovial fluid $(p<0.01)$ taken from patients with rheumatoid arthritis compared with samples taken fron non-RA patients. The highest CL max responses were produced by synovial fluid obtained from 2 patients with Felty's syndrome and 5 patients with severe active RA. Retrospective comparative studies of these results with assays of $\mathrm{Clq}$ binding for immune complexes and rheumatoid factor showed no significant correlation.

As large quantities of an inflammatory pleural fluid were available from a patient with probable RA, this specimen was studied in more detail. This fluid gave a high CL max level (value $=315$ ), contained large quantities of immune complexes, and had greatly depressed levels of $\mathrm{C} 3$ and $\mathrm{C} 4$. The $\mathrm{CL}$ reactive material precipitated when the specimen was incubated either at $4{ }^{\circ} \mathrm{C}$ overnight or with $3 \%$ polyethylene glycol (PEG). This fluid was fractionated by 


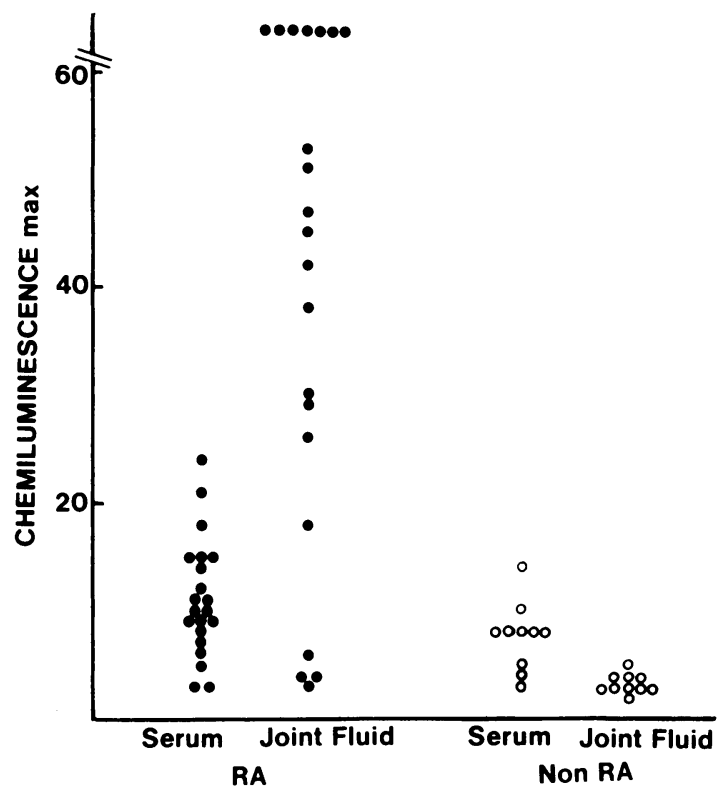

Fig. 1 Neutrophil CL max values (expressed in millivolts) for paired samples of serum and synovial fluid taken from 21 patients with classical or definite $R A$ and 10 patients with other joint diseases. The seven $C L$ max values $>60$ for knee synovial fluid varied over the range 70-600 and were obtained from patients with Felty's syndrome (recording levels of 107 and 240 respectively) and 5 patients with severe rheumatoid disease.

Sepharose 6B filtration chromatography and the column fractions tested for CL activity and IgG concentration. The peak CL activity was found in column fractions containing large molecular weight $\mathrm{IgG}$ (MW $>4 \times 10^{6}$ daltons), while a small region of activity was seen in fractions eluting in the monomeric IgG position (Fig. 2).

Preparations of HAGG also induced neutrophil $\mathrm{CL}$ activity. Testing HAGG which had been prepared by heating at $63^{\circ} \mathrm{C}$ for varying lengths of time indicated that a minimum heating time of 45-60 min was required before the preparations were capable of inducing chemiluminescence. Aggregates induced by heating for shorter periods of time appeared to be poorly or nonreactive. After filtration chromatography of HAGG, fractions were tested, and CL was again found to be restricted to those aggregates of large molecular size ( $\mathrm{MW}>10^{6}$ daltons) (Fig. 3).

A monoclonal rheumatoid factor with strong avidity for aggregated IgG was preincubated with sera from samples and controls. These preincubated samples showed increases in CL activity greater than 10 times the preincubation levels (Fig. 4). Similar

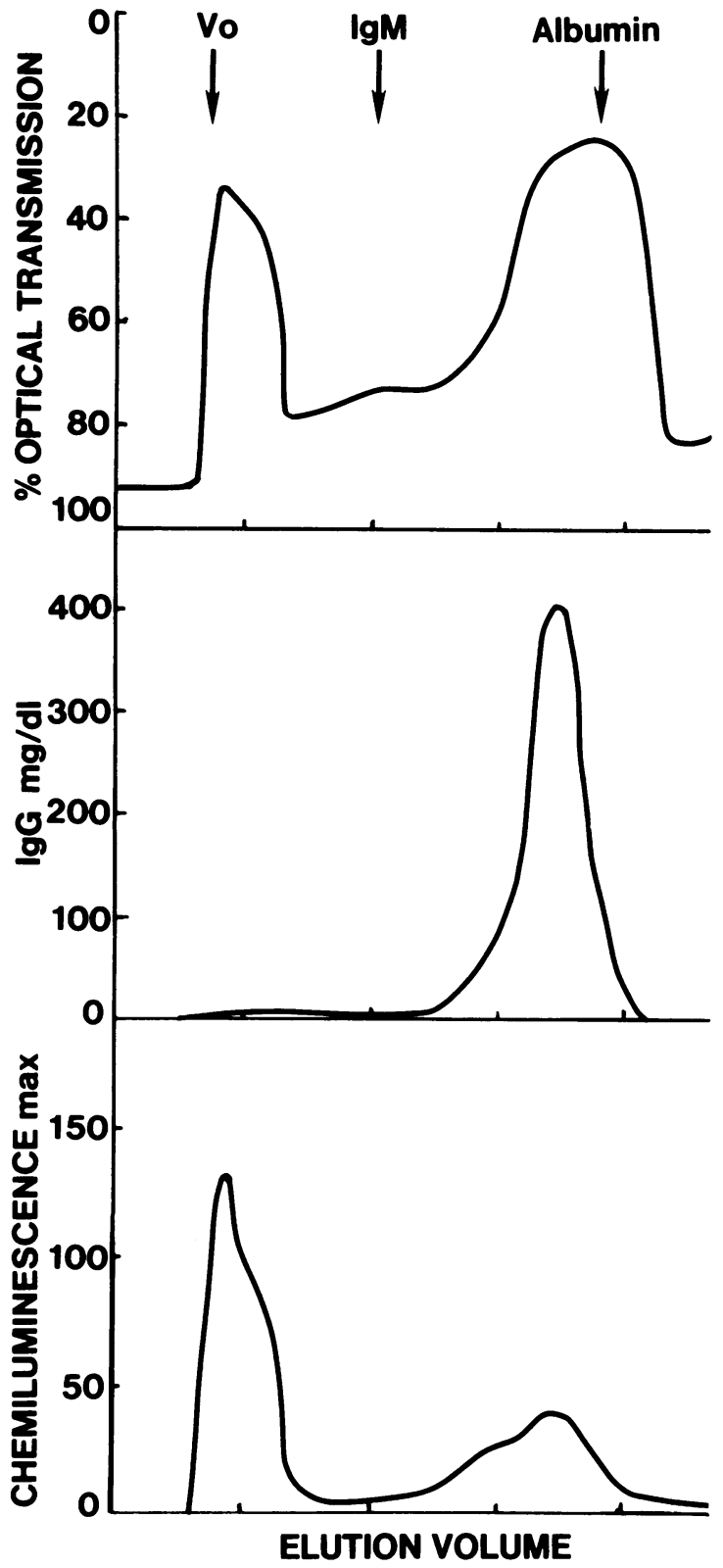

Fig. 2 Upper panel: The elution profile (\% optical transmission) following sepharose $6 B$ filtration chromatography of 5 ml of pleural fluid. The elution position of the void volume $\left(V_{0}\right), \operatorname{IgM}$, and albumin are indicated. Middle panel: The IgG concentration profile of the same column fractions showing a large shelf of complexed $\mathrm{IgG}$ extending into the void volume. Lower panel: The CL max values obtained when the column fractions were incubated with normal neutrophils. Two peaks of activity coinciding with the fractions containing large molecular weight complexed $\lg G$ and monomeric $\lg G$ are demonstrated. 


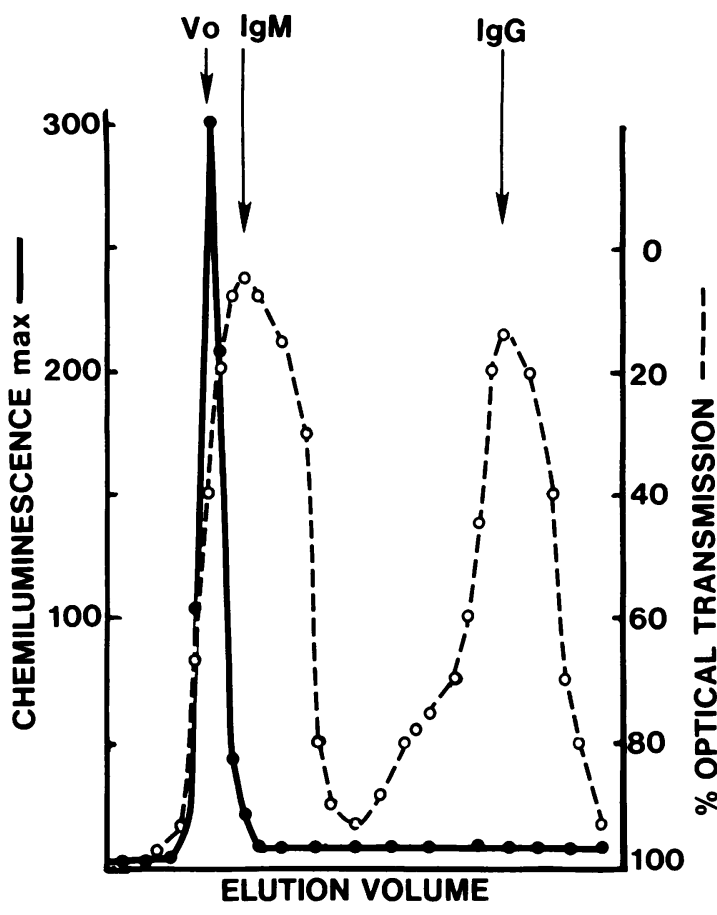

Fig. 3 The elution profile of a $H A G G$ preparation after filtration chromatography on Sephacryl S300 $0---0$. The $C L$ max readings obtained after incubating the column fractions with normal neutrophils are indicated - -0 . The elution position of $\operatorname{IgM}$, monomeric $\operatorname{IgG}$, and the void volume $\left(V_{0}\right)$ are shown. increases in CL activity were observed when preparations of HAGG which had previously been nonreactive or poorly reactive were preincubated with $\mathrm{mRF}$. The monoclonal reagent itself did not induce CL.

Indirect fluorescent examination of neutrophils preincubated in $C L$ reactive sera or synovial fluid showed intracellular inclusions of immunoglobulin in more than $90 \%$ of the neutrophils. Less than $5 \%$ of the neutrophils incubated in nonreactive control sera showed positive staining. The antiserum used contained only $F(a b)_{2}^{1}$ fragments, thereby avoiding the possibility of nonspecific staining of the neutrophil surface by binding to Fc receptors.

\section{Discussion}

The detection of a CL response when normal neutrophils were preincubated in samples taken from patients with RA is direct evidence of neutrophil activation by those specimens. Particularly strong activation was observed with specimens of synovial fluid obtained from patients with severe RA or Felty's syndrome and with a single inflammatory pleural fluid from a patient with probable RA. These strong responses may reflect the site of highest concentration of the reactive material and hence the site of neutrophil activation in vivo.

Detailed study of the single pleural fluid provided evidence relating to the nature of the reactive
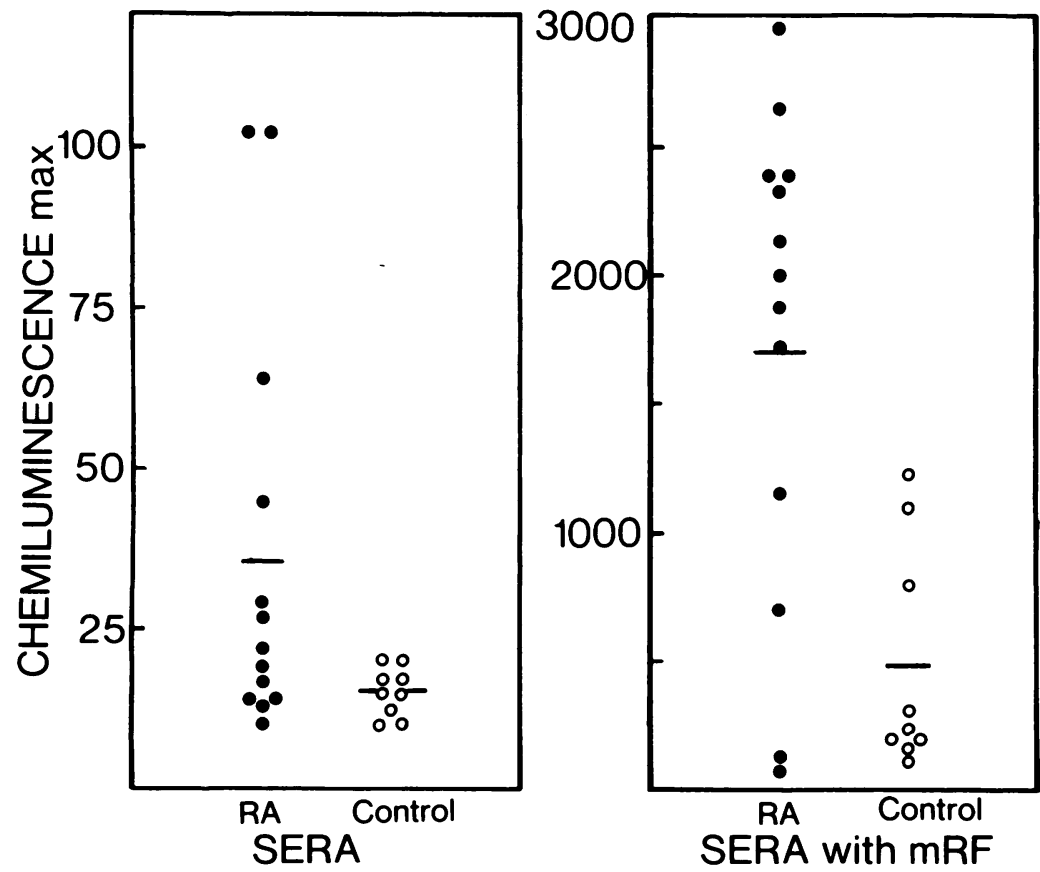

Fig. 4 Neutrophil CL max values observed following incubation of normal neutrophils in 13 RA and 9 control sera and the samples after preincubation with $m R F$. 
material. Cold and PEG induced precipitation and column filtration studies suggested that the reactive material contained complexed IgG of large molecular weight. In addition a smaller CL peak was found eluting in the column fractions containing monomeric IgG. The nature of the reactive material in this low molecular weight region is unclear but might suggest a functionally active antineutrophil antibody. These observations may help to explain the lack of correlation noted between the CL max responses produced by the sera and synovial fluids and the amount of immune complexes as measured by the $\mathrm{C} 1 \mathrm{q}$ binding method and levels of rheumatoid factor. This lack of correlation suggests that the immune complexes causing neutrophil activation may be different from those previously described in RA, which are complement fixing and involve both IgG and IgM rheumatoid factor. ${ }^{10}$

The CL activity produced by pathological samples was mimicked by suspensions of HAGG which were used as a model of immune complexes. Column chromatographic analysis of these preparations and the prolonged heating time required for the production of these reactive aggregates support the concept that large molecular sized IgG aggregates are responsible for producing a $\mathrm{CL}$ reaction.

Further indirect evidence that complex size is important in determining CL reactivity was obtained using a mRF which had a strong avidity for complexed IgG. The addition of this reagent caused a dramatically amplified CL response. In addition the indirect immunofluorescent studies with an antiserum directed against human immunoglobulin provide further evidence that the reactive material contains immunoglobulin. Fluorescent microscopy suggested that a correlation exists between the intensity of intracellular staining of the immunoglobulin inclusions in neutrophils and levels of CL reactivity produced by the same sera (unpublished). This area is being further investigated.

The levels of $C L$ induced by the more reactive rheumatoid specimens as demonstrated in this study are comparable with the levels of CL produced during neutrophil phagocytosis of opsinised zymosan (unpublished). This suggests that the phenomenon is of some biological importance. Neutrophil $\mathrm{CL}$ is a measure of the generation of superoxide and other $\mathrm{O}_{2}$ free radicals which accompany phagocytosis. ${ }^{11}$ These high-energy metabolically reactive radicals have been strongly implicated as causative agents in the process of tissue damage.$^{12}$ We propose that the direct neutrophil activation induced by immune complex containing rheumatoid sera and synovial and pleural fluid in vitro may also occur in vivo and as such have an important role in the immunopathogenesis of the inflammatory rheumatoid process.

Note added in proof: Since submission of this paper we have noted similar findings by Starkebaum et al. ${ }^{13}$

We thank Mrs M. Molnar for expert secretarial assistance and for typing the script. J. V. Bertouch is a greatful recipient of an $\mathrm{NH}$ and MRC postgraduate scholarship.

\section{References}

1 Ziff M. Autoimmune processes in rheumatoid arthritis. Prog Immunol 1974; 5: 37-46.

2 Zubler R H, Nydegger U, Perrin L $\mathrm{H}$, et al. Circulating and intra-articular immune complexes in patients with rheumatoid arthritis. J Clin Invest 1976; 57: 1308-19.

3 Hurd E R, Andreis M, Ziff M. Phagocytosis of immune complexes by polymorphonuclear leucocytes in patients with Felty's syndrome. Clin Exp Immunol 1977; 28: 413-25.

4 Howe G B, Fordham J N, Brown K A, Currey H L F. Polymorphonuclear cell function in rheumatoid arthritis and in Felty's syndrome. Ann Rheum Dis 1982; 40: 370-5.

5 Ugae K, Ziff M, Jasin H E. Interaction of polymorphonuclear leukocytes with immune complexes trapped in joint collagenous tissues. Arthritis Rheum 1979; 22: 353-64.

6 Hashimoto Y, Hurd E R. Human neutrophil aggregation and increased adherence to human endothelial cells induced by heat-aggregated $\mathrm{IgG}$ and immune complexes. Clin Exp Immunol 1981; 44: 538-47.

7 Doll N J, Wilson M R, Salvaggio J E. Inhibition of polymorphonuclear leukocyte chemiluminescence for detection of immune complexes in human sera. J Clin Invest 1980; 66: 457-64.

8 Roberts-Thomson P J, Bradley J. A nephelometric study of the reaction of monoclonal rheumatoid factor with heat aggregated gammaglobulin and sera from patients with immune complex diseases. Clin Exp Immunol 1979; 37: 408-15.

9 Roberts-Thomson P J, Neoh S H, Bradley J, Milazzo S C. Circulating and intra-articular immune complexes in rheumatoid arthritis: a comparative study of the C1q binding and monoclonal rheumatoid factor assays. Ann Rheum Dis 1980; 39: 438-44.

10 Male D, Roitt I M, Hay F C. Analysis of immune complexes in synovial effusions of patients with rheumatoid arthritis. Clin Exp Immunol 1980; 39: 297-306.

11 Schadelin J, Schadelin R, Mandell G L. Chemiluminescence of phagocytic cells. CRC Crit Rev Clin Lab Sci 1980; 13: 1-19.

12 Klebanoff $S \mathrm{~J}$. Oxygen metabolism and the toxic properties of phagocytes. Basic Review. Ann Intern Med 1980; 93: 480-9.

13 Starkebaum G, Stevens D L, Henry C, Gavin S E. Stimulation of human neutrophil chemiluminescence by soluble immune complexes and antibodies to neutrophils. J Lab Clin Med 1981; 98: 280-91. 